\title{
High-resolution spectroscopy of the $\delta$ Scuti star 44 Tauri: photospheric element abundances and mode identification ${ }^{\star}$
}

\author{
W. Zima ${ }^{1,2}$, H. Lehmann ${ }^{3}$, Ch. Stütz ${ }^{2}$, I. V. Ilyin ${ }^{4}$, and M. Breger ${ }^{2}$ \\ 1 Instituut voor Sterrenkunde, K.U. Leuven, Celestijnenlaan 200D, 3001 Leuven, Belgium \\ e-mail: zima@ster.kuleuven.be \\ 2 Institut für Astronomie, Universität Wien, Türkenschanzstrasse 17, 1180 Vienna, Austria \\ Thüringer Landessternwarte, 07778 Tautenburg, Germany \\ 4 Astrophysikalisches Institut Potsdam, An der Sternwarte 16, 14482 Potsdam, Germany
}

Received 31 January 2007 / Accepted 28 May 2007

\section{ABSTRACT}

\begin{abstract}
Aims. We carried out a detailed spectroscopic study of the $\delta$ Sct star 44 Tau to investigate its element abundances and stellar parameters as well as to provide an identification of its non-radial oscillation modes.

Methods. We acquired high-resolution high-signal-to-noise ratio time-series spectroscopy of 44 Tau from two observing sites in Europe during a time span of 30 days. We determined atmospheric element abundances and stellar properties using spectroscopic methods. Due to the low value of $v \sin i$ the mode identification was carried out by applying the moment method.

Results. A very low value of the projected rotational velocity of $v \sin i=2 \pm 1 \mathrm{~km} \mathrm{~s}^{-1}$ was determined. We detected spectroscopically 12 independent frequencies between 6.8 and $11.6 \mathrm{~d}^{-1}$ and four combination frequencies by analyzing the line moments as well as the pixel-intensities across the profiles of carefully selected Fe absorption lines. The mode identification applying the moment method yielded six axisymmetric pulsation modes. Two frequencies are low-degree prograde pulsation modes. A rather low value of $\log g=3.6$ was determined implying an evolved evolutionary status of 44 Tau. The derived atmospheric element abundances are close to solar.

Conclusions. Our analysis revealed that 44 Tau is an exceptionally slowly rotating $\delta$ Scuti star pulsating with a rich spectrum of axisymmetric pulsation modes. This simplifies the seismic modeling since rotational perturbations of higher order can be neglected.
\end{abstract}

Key words. techniques: spectroscopic- line: profiles - stars: abundances - stars: oscillations - stars: variables: $\delta$ Scuti stars: individual: HD26322 = 44 Tau

\section{Introduction}

Asteroseismology allows the study of the interior structure of oscillating stars. The stellar oscillations lead to periodic flux and velocity variations of the photosphere, which depend on the physical conditions of its interior. The aim of asteroseismology is to reproduce the measured variations by an appropriate physical model which precisely describes the interior structure of the star. Multi-periodic non-radial oscillations are ideally suited for asteroseismology, since different pulsation modes propagate in different layers inside the star. Such pulsations are characterized by their frequencies and three quantum numbers, the radial order $n$, the spherical degree, $\ell$, and the azimuthal order, $m$.

Seismic stellar models predict that evolved $\delta$ Sct stars pulsate simultaneously with hundreds of low-degree modes. This is due to the fact that high-order gravity modes, which propagate in the deep interior, penetrate the pressure mode frequency domain as a result of the increasing density of the stellar core. Thus, the observation of such mixed modes yield important information about the regions close to the core. The presently applied linear pulsation theory cannot predict pulsation amplitudes. Since only a fraction of the theoretically predicted pulsation frequencies is observed due to the generally low photometric amplitudes

* Based on observations partly collected at the Thüringer Landessternwarte Tautenburg (TLS) and at the Nordic Optical Telescope (NOT) at La Palma. of non-radial pulsation modes and an unknown mode selection mechanism, a simple comparison between measured and theoretical frequencies has not been successful for this type of pulsating stars. Successful seismic modeling thus relies on the precise determination of the pulsation frequencies and on the identification of their quantum numbers. Mode identification is especially important for $\delta$ Sct stars, which pulsate in a multitude of low radial-order modes far below the asymptotic limit.

It has been demonstrated for the case of FG Vir by Breger et al. (2005), Zima et al. (2006) and Daszynska-Daszkiewicz et al. (2005) that for slowly rotating $\delta$ Sct stars, which pulsate non-radially with modes of low-radial order, different complementory methological approaches are required to determine all three quantum numbers, $\ell, m$ and the radial order, $n$. Whereas the amplitude ratio and phase difference obtained from photometric measurements in different filters are best suited for the determination of $\ell$, spectroscopy is needed to derive the azimuthal order $m$. The radial order $n$ can only be determined when confronting the observed frequency values with theoretical predictions, which generally requires a reliable identification of the other two quantum numbers beforehand.

Spectroscopy provides a tool to determine the pulsation geometry of stars by the precise measurement of intensity variations in the profile of absorption lines. Presently, it is the only known way to determine simultaneously the $(\ell, m)$-values of pulsation modes. The following spectroscopic methods permit the identification of $\ell$ and $m$, the intrinsic pulsation amplitude 
and the stellar inclination angle and have been successfully applied to various kinds of non-radially pulsating stars: the moment method (Balona 1986; Aerts 1996; Briquet \& Aerts 2003), the intensity-period-search method (Schrijvers et al. 1997), the pixel-by-pixel method (Mantegazza 2000) and the Fourier parameter fit method (Zima 2006).

By means of the moment method, the first three line moments $\left(\left\langle v^{1}\right\rangle,\left\langle v^{2}\right\rangle,\left\langle v^{3}\right\rangle\right)$, measures for the radial velocity, the line width, and the line skewness, respectively, are fitted with theoretical values. It is optimally suited for the analysis of slowly rotating stars having a rotational velocity below $100 \mathrm{~km} \mathrm{~s}^{-1}$. Its application is presently limited to the identification of pulsation modes up to a degree $\ell$ of 4 . The three other methods all rely on the Doppler broadening of the line profiles. Basically, for these methods there is no upper application limit in terms of $\ell$ or $m$ and for some fast rotating objects high-degree modes have been reported (e.g., Mantegazza 2004; Schrijvers \& Telting 2002). Currently, only the Fourier parameter fit method allows a statistical determination of the significance of the obtained identifications.

The star 44 Tau belongs to the class of $\delta$ Sct stars (for a detailed review see, e.g., Breger 2000). Its photometric variability was discovered by Danziger \& Dickens (1967) who classified it as a $\delta$ Sct star. Subsequent photometric observations of this object by Percy (1973), Desikachary (1973) and Lopez de Coca et al. (1987) revealed this star to be multi-periodic having periods around $3 \mathrm{~h}$. From 119 hours of photometry, Poretti et al. (1992) reported the detection of seven frequencies between 6.89 and $11.52 \mathrm{~d}^{-1}$. They identified the frequencies at $6.89,8.96$ and $11.52 \mathrm{~d}^{-1}$ as radial fundamental and its first two overtones. A very low inclination angle of $11^{\circ}$ was determined by assuming that some of the observed frequencies are rotationally split.

The first spectroscopic monitoring of this star was carried out by Smith (1982). From five high-resolution spectra $(R=50000)$ centered on the Fe I and the Fe II lines at $\lambda 5408 \AA$, he estimated a semi-amplitude of the radial velocity of $1.9 \mathrm{~km} \mathrm{~s}^{-1}$ for the dominant mode $\left(6.89 \mathrm{~d}^{-1}\right)$. Moreover, an exceptionally low projected rotational velocity $v \sin i=5 \mathrm{~km} \mathrm{~s}^{-1}$ was determined. Thus, 44 Tau is one of the slowest rotating known $\delta$ Sct stars. This is quite unusual since slow rotation in $\delta$ Sct stars is generally associated with radial pulsation having relatively high amplitudes of a few tenths of a magnitude, whereas 44 Tau pulsates with a significantly lower amplitude. The low $v \sin i$ was confirmed by Solano \& Fernley (1997) who determined a value of $6.8 \pm 1.2 \mathrm{~km} \mathrm{~s}^{-1}$. Intrinsically slow rotation and its multi-periodic non-radial pulsations made 44 Tau a very promising target from a theoretical point of view, since rotationally split multiplets would be easily recognizable in the frequency spectrum.

During 2000 through 2003 the Delta Scuti Network (see Rodler et al. 2003) acquired $470 \mathrm{~h}$ of photometry of 44 Tau in the Strömgren filters $v$ and $y$ (Antoci et al. 2007, hereafter A07). Their data revealed 29 frequencies: 13 independent frequencies between 6.33 and $12.69 \mathrm{~d}^{-1}$ and 16 combination frequencies (harmonics $2 f_{i}$ and sums $f_{i}+f_{j}$ ). The amplitudes of the detected frequencies are from about $40 \mathrm{mmag}$ in $v$ down to $0.37 \mathrm{mmag}$. A07 report the identification of the radial fundamental $\left(6.89 \mathrm{~d}^{-1}\right)$ and its first overtone $\left(8.96 \mathrm{~d}^{-1}\right)$ from their frequency ratio of 0.7698 and by comparing observed and theoretical Q-values. Also the positive phase difference in the $v$ and $y$ passbands support this. Such an identification sets strong constraints for the seismic modeling.

In this paper we describe the detailed analysis of new spectroscopic observations we have acquired for 44 Tau. Section 2 describes this new set of data. In the following section, the
Table 1. Log of spectroscopic observations of 44 Tau. The heliocentric start and end date are given in the first two columns followed by the observing duration in hours, the number of acquired spectra and the observing site.

\begin{tabular}{ccccc}
\hline \hline \multicolumn{2}{c}{ HJD +2450000} & $\begin{array}{c}\text { Length } \\
\text { Begin }\end{array}$ & $\begin{array}{c}\text { No. of } \\
\text { End }\end{array}$ & Obs. \\
\hline 3331.2439 & 3331.3420 & 2.35 & 13 & TLS \\
3333.2740 & 3333.5435 & 6.47 & 31 & TLS \\
3334.2199 & 3334.3857 & 3.98 & 42 & TLS \\
3334.5948 & 3334.7017 & 2.56 & 11 & TLS \\
3335.2403 & 3335.6783 & 10.51 & 93 & TLS \\
3335.3890 & 3335.5504 & 3.87 & 18 & NOT \\
3336.3581 & 3336.7335 & 9.01 & 45 & NOT \\
3337.4119 & 3337.7268 & 7.56 & 37 & NOT \\
3343.4593 & 3343.5604 & 2.43 & 11 & TLS \\
3360.2050 & 3360.6353 & 10.33 & 72 & TLS \\
3361.2090 & 3361.6432 & 10.42 & 70 & TLS \\
\hline
\end{tabular}

atmospheric properties and abundances are reported. Finally, in Sect. 4 we present the frequency analysis and mode identification.

\section{Observations}

The detailed study of profile variations of absorption lines requires time-series spectra of high-SNR and high resolution $(R>$ $50000)$. High resolution was of utmost importance for our target as it is known to have a very low $v \sin i$.

We acquired spectroscopic measurements of 44 Tau at two European sites during November and December 2004. In total, 443 spectra were obtained covering $69.49 \mathrm{~h}$. A journal of the observations is given in Table 1.

The observations at the Thüringer Landessternwarte Tautenburg (TLS, Germany) were obtained with the $2 \mathrm{~m}$ telescope equipped with a Coudé Echelle spectrograph. The wavelength range was 4708-7200 $\AA$ at a resolution of $R=63000$. Integration times were between 2.5 and 10 min depending on the seeing conditions. The observer was Holger Lehmann. The TLS spectra were reduced by using standard MIDAS reduction packages including filtering of cosmic rays, bias and stray-light subtraction, flat fielding, optimized order extraction, and normalization to the local continuum. In addition to the wavelength calibration by Th-Ar-lamp spectra, an individual zero-point correction was applied to every spectrum by using the positions of a large number of telluric $\mathrm{O}_{2}$ lines.

The measurements at the Nordic Optical Telescope (NOT, La Palma, Spain) were carried out with the SOFIN spectrograph attached to the $2.5 \mathrm{~m}$ telescope. We observed at a resolution of $R=80000$ covering a wavelength range between 5000 and $7000 \AA$. The integration time was $10 \mathrm{~min}$. This is about $5 \%$ of the shortest known pulsation period of 44 Tau and thus short enough to avoid significant phase smearing. The observers were Ilya Ilyin and Wolfgang Zima. The Echelle spectra were reduced with the software package described in Ilyin (2000). This includes bias subtraction, master flat field correction, scattered light subtraction approximated with bi-cubic splines, weighted extraction of spectra, and accurate wavelength calibration with the use of two Th-Ar spectra taken before and after each object exposure. Continuum rectification and CCD fringes removal was done with the flat field spectra reduced in the same manner.

The theoretical frequency resolution that can be achieved from the time sampling of our data is $0.05 \mathrm{~d}^{-1}$ (Loumos \& Deeming 1978). 

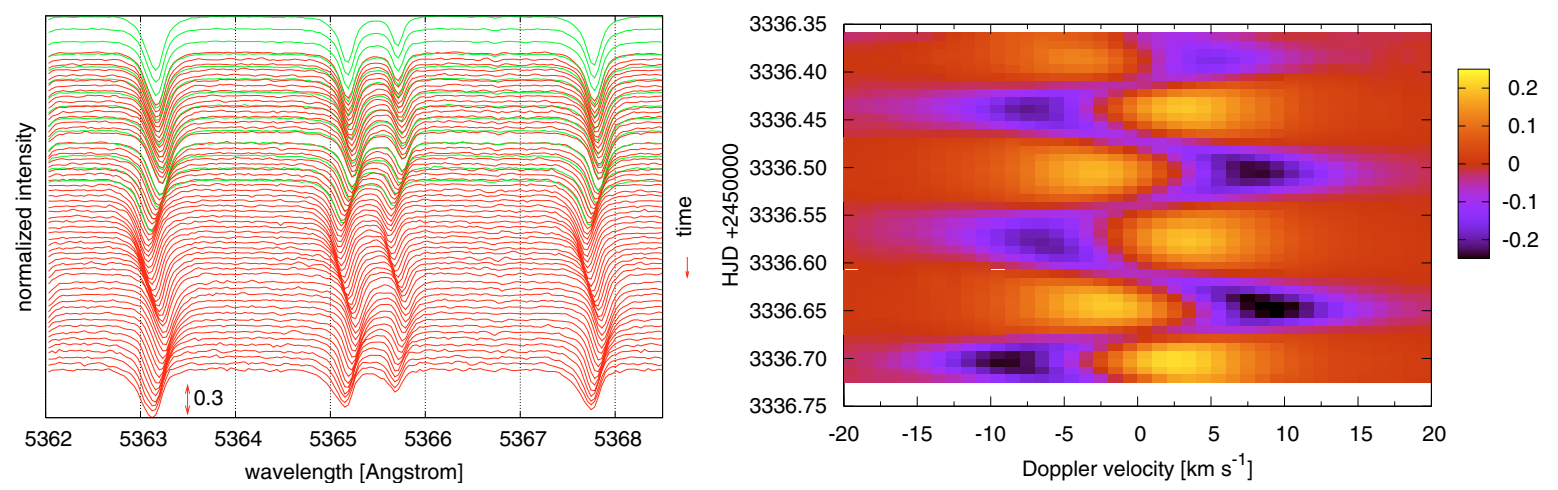

Fig. 1. Line profile variations due to the oscillations of 44 Tau. Left panel: wavelength range between 5362 and $5368 \AA$ measured at the NOT and at TLS during 2004 Nov. 26/27. For a better representation, spectra taken at consecutive times are shifted vertically. Right panel: deviations of the Fe I $15383.369 \AA$ line from the mean intensity during 2004 Nov. $27 / 28$ at the NOT.

\section{Atmospheric parameters}

The amplitude of the radial velocity variations of 44 Tau is more than $2 \mathrm{~km} \mathrm{~s}^{-1}$, which is of the order of the projected rotational velocity of this star. Therefore, a spectrum integrated for a time longer than a few percent of the period of the dominant pulsation mode would cause artificial line broadening. Our spectra were integrated between 2.5 and $10 \mathrm{~min}$, which is only a fraction of the pulsation period. Furthermore, a single spectrum is not well suited for an abundance analysis due to the fact that the line profiles are strongly distorted by the stellar oscillations. To compute a spectrum which most closely resembles the non-pulsating case we selected four spectra measured at TLS which all have an absolute value of the first moment of below $0.02 \mathrm{~km} \mathrm{~s}^{-1}$ and computed a mean of these spectra for the further analysis. The nominal resolution of these spectra was $R=63000$. A check with atmospheric lines around $6280 \AA$ yielded $R=65000$ which was assumed for the following analysis.

We derived starting values for the fundamental atmospheric parameters by using the latest measurements from Strömgren $u v b y \mathrm{H}_{\beta}$ photometry (Hauck \& Mermilliod 1998). The application of the program TEMPLOGG.V2 (Rogers 1995) yielded $T_{\text {eff }}=6900 \mathrm{~K}$ and $\log g=3.57$. The observed line profiles of $\mathrm{H}_{\alpha}$ and $\mathrm{H}_{\beta}$ were compared to synthesized profiles and resulted in $T_{\text {eff }}=6700 \mathrm{~K}$ and $\log g=3.5$. But since the hydrogen lines cover several Echelle orders, the fit to the continuum is very uncertain in these regions. Thus, we started our abundance analysis by adopting the fundamental parameters derived by photometry. Due to the interdependency of the photospheric parameters $T_{\mathrm{eff}}$, $\log g, v_{\text {micro }}$, element abundances, and magnetic field strength, these values had to be refined iteratively.

For our analysis we used the wavelength range between 4720 and $6720 \AA$. The adopted spectrum had a signal-to-noise ratio (SNR) of about 300. We computed a grid of atmospheric models using ATLAS9 (Kurucz 1993) and LLmodels (Shulyak et al. 2004) for the following parameter range [min, max, step]: $T_{\text {eff }}[6500,7000,50], \log g[3.5,4.0,0.1], v_{\text {micro }}[0,5,1]$. The wavelength values of the absorption lines were extracted from VALD (Piskunov et al. 1995; Kupka et al. 1999; Ryabchikova et al. 1999). Line synthesis was performed with synth3 (Kochukhov 2006).

\subsection{Equivalent widths}

We measured the equivalent widths of 132 unblended or nearly unblended metallic lines of various species. Using lines

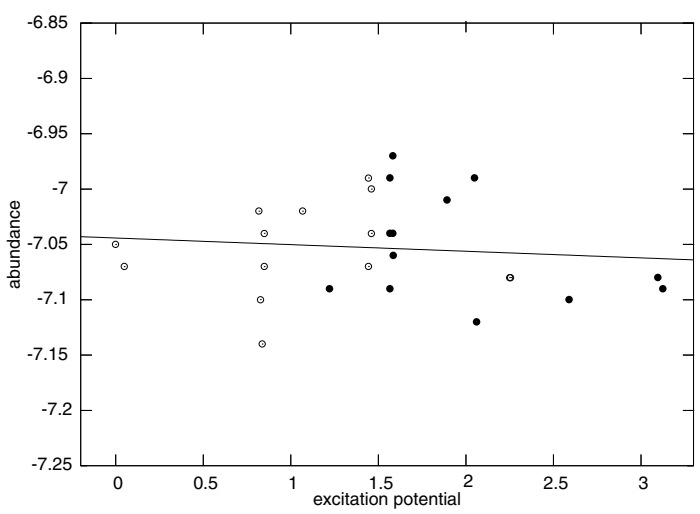

Fig. 2. Abundance versus excitation potential for Ti I and Ti II for $T_{\text {eff }}=7000 \mathrm{~K}$ and $\log g=3.6$. Open circles are Ti I lines, filled circles Ti II. The correlation is nearly zero and ionization balance is fulfilled. Abundances are given in $\log \left(N_{\text {elm }} / N_{\text {tot }}\right)$.

of Fe I, Fe II, Ni I and Mn I we determined a microturbulence of $v_{\text {micro }}=1.85 \mathrm{~km} \mathrm{~s}^{-1}$. To determine $T_{\text {eff }}$ and $\log g$, abundances from lines of $\mathrm{Fe}, \mathrm{Ti}, \mathrm{Ni}$ and $\mathrm{Mn}$ were compared to their respective excitation potentials. By assuming LTE, no correlation between the excitation potentials and the abundances should exist for models with the correct effective temperature. In an iterative way we derived an effective temperature of $7000 \pm 200 \mathrm{~K}$, a surface gravity of $3.6 \pm 0.1$ and abundances for 9 elements. If our assumption of ionization balance holds, the abundances derived from lines of different ionization stages of the same element (in our case $\mathrm{Fe}$ and $\mathrm{Ti}$ ) should be equal. Figure 2 demonstrates this for the final values of $T_{\text {eff }}$ and $\log g$.

\subsection{Line synthesis}

The values of $v \sin i$ and the macro turbulence $v_{\text {macro }}$ were determined from $14 \mathrm{Fe}$ I lines spread over the whole range of the spectrum. For the projected rotation velocity we can only give an upper limit of $v \sin i<3 \mathrm{~km} \mathrm{~s}^{-1}$ (see Fig. 3). Our best fit yielded a value of $2 \mathrm{~km} \mathrm{~s}^{-1}$, which we used for spectrum synthesis. The wings of deep lines are stronger than what we would expect from their depth and our value for the macro turbulence of $v_{\text {macro }}=2 \mathrm{~km} \mathrm{~s}^{-1}$.

Table 2 lists the abundance pattern we determined for 44 Tau by means of spectrum synthesis. The derived abundances are similar to solar values. The elements $\mathrm{Ca}, \mathrm{Co}, \mathrm{Mg}$, and $\mathrm{Mn}$ seem 


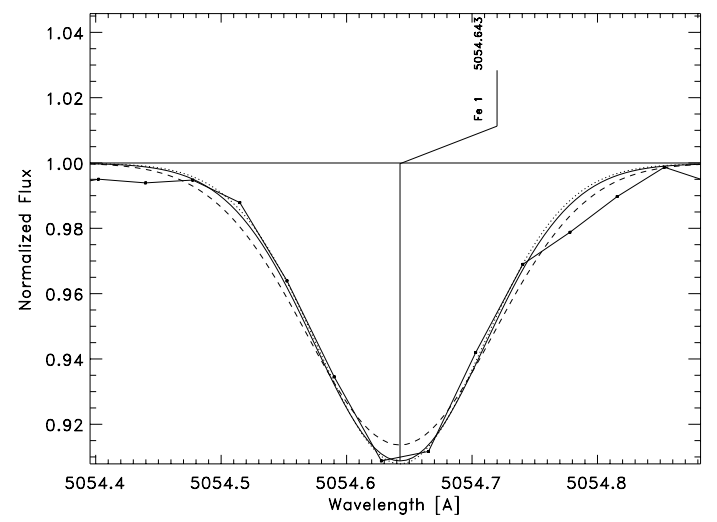

Fig. 3. Synthesis of Fe I $\lambda 5054.643 \AA$ for 3 different values of $v \sin i$. The solid line represents our adopted value of $2 \mathrm{~km} \mathrm{~s}^{-1}$, the dotted line $0 \mathrm{~km} \mathrm{~s}^{-1}$ and the dashed line the already too high $v \sin i$ of $4 \mathrm{~km} \mathrm{~s}^{-1}$.

Table 2. Mean element abundances (abn) and uncertainties $\left(\sigma_{\mathrm{abn}}\right)$ of 44 Tau determined from line synthesis. The solar abundances (Col. 5) were taken from Grevesse \& Sauval (1998). The difference $(\Delta)$ between the abundances of 44 Tau and the sun are listed in Col. 6. All element abundances are indicated in units of $\log \left(N / N_{\text {tot }}\right)$.

\begin{tabular}{cccccc}
\hline \hline Element & \# lines & abn & $\sigma_{\text {abn }}$ & $\mathrm{abn}_{\odot}$ & $\Delta$ \\
\hline $\mathrm{Ca}$ & 17 & -5.53 & 0.07 & -5.68 & 0.15 \\
$\mathrm{Co}$ & 7 & -6.83 & 0.08 & -7.12 & 0.29 \\
$\mathrm{Cr}$ & 25 & -6.25 & 0.09 & -6.37 & 0.12 \\
$\mathrm{Fe}$ & 244 & -4.45 & 0.13 & -4.54 & 0.09 \\
$\mathrm{Mg}$ & 1 & -4.32 & - & -4.46 & 0.14 \\
$\mathrm{Mn}$ & 11 & -6.44 & 0.14 & -6.65 & 0.21 \\
$\mathrm{Ni}$ & 52 & -5.80 & 0.12 & -5.79 & -0.01 \\
$\mathrm{O}$ & 2 & -3.41 & 0.01 & -3.21 & -0.20 \\
$\mathrm{Sc}$ & 4 & -9.24 & 0.08 & -8.87 & -0.37 \\
$\mathrm{Ti}$ & 27 & -7.05 & 0.04 & -7.02 & -0.03 \\
$\mathrm{~V}$ & 4 & -7.98 & 0.05 & -8.04 & 0.06 \\
\hline
\end{tabular}

Table 3. Fundamental parameters of 44 Tau derived from photometric calibrations (Col. 2), line equivalent widths (Col. 3) and line synthesis (Col. 4).

\begin{tabular}{lrrr}
\hline \hline & phot. & EQW & line synthesis \\
\hline$T_{\text {eff }}[\mathrm{K}]$ & 6900 & 7000 & 7000 \\
$\log g$ & 3.57 & 3.60 & 3.60 \\
$v_{\text {micro }}\left[\mathrm{km} \mathrm{s}^{-1}\right]$ & - & 1.85 & 1.30 \\
$v \sin i\left[\mathrm{~km} \mathrm{~s}^{-1}\right]$ & - & - & $<3$ \\
$v_{\text {macro }}\left[\mathrm{km} \mathrm{s}^{-1}\right]$ & - & - & 2 \\
\hline
\end{tabular}

to be slightly overabundant, whereas $\mathrm{O}$ and $\mathrm{Sc}$ appear to be underabundant.

No sign for a global magnetic field could be detected by looking for magnetically split lines and by checking the relation between abundances and Landé factors. From our data we can exclude a magnetic field strength higher than $2 \mathrm{kG}$. The final set of atmospheric parameters resulting from our findings from photometry, the analysis of the equivalent widths and the line synthesis is summarized in Table 3.

\section{Frequency detection and mode identification}

\subsection{Frequency detection}

We computed a combined profile for further analysis in the following way. First we selected five unblended absorption lines of neutral iron. The condition that the lines originate from the same element and ionization state is important to avoid different behavior of the individual lines during the pulsation cycle. We chose the following lines after careful inspection of the spectra: Fe I $\lambda 5367.467 \AA$, Fe I $\lambda 5373.709 \AA$, Fe I $\lambda 5383.369 \AA$, Fe I $\lambda 5393.168 \AA$, and Fe I $\lambda 5398.279 \AA$.

For each line a separate Fourier analysis of the first moment and of the intensity variations across the profile was carried out. Since the first moment amplitudes and their phases showed coherent behavior in all selected lines, we accepted them to be safe for combining. The center of each line was determined separately by fitting a synthetic rotationally broadened Gaussian profile to the observed zero point profile. The latter was computed from a two-dimensional least-squares fit for each wavelength bin across the line by fitting a sum of sinusoidals. The wavelength values of each line were converted to Doppler velocities relative to the determined line centers and a mean spectrum was computed for further analysis. This resulting spectrum had a SNR of about 420, which is an appropriate value for carrying out spectroscopic mode identification.

The mean velocity of 44 Tau relative to the barycenter of the solar system is, according to the shift of the five selected lines, $+17.5 \pm 0.1 \mathrm{~km} \mathrm{~s}^{-1}$. This coincides perfectly with the values derived by Smith (1982) and by Nordstroem et al. (2004).

We searched for periodicities in the combined spectrum by analyzing the line moments and the intensity variations of the individual wavelength bins across the line. Whereas the moments are generally better suited for the detection of low degree modes $(\ell \leq 3)$, the latter are also sensitive to high-degree pulsation modes. Since the projected rotational velocity of 44 Tau is so low we can expect to find in both cases the same low-degree pulsation modes as in photometry.

The frequency search was carried out with discrete Fourier transformation and non-linear least-squares fitting techniques using Period04 (Lenz \& Breger 2005) for the one-dimensional time-series and own codes for the two-dimensional analyses. By the least-squares optimization a sum of $N$ sinusoidals is fitted to the data using the formalism $Z+\sum_{i=1}^{N} A_{i} \sin \left(2 \pi f_{i}+\phi_{i}\right)$, where $Z$ denotes the zero point, $A_{i}$ is the amplitude of the frequency $f_{i}$, and $\phi_{i}$ is its phase. Iteratively, the data were pre-whitened with all detected frequencies plus the frequency having highest amplitude in the Fourier spectrum. As a significance limit for the detection of new frequencies we used the criterion $S N R=4$ in amplitude (Breger et al. 1999), and $S N R=3.5$ for combination frequencies $\left(f_{i} \pm f_{j}\right)$. The photometric observations by A07 provide a frequency precision which is better by a factor of about 30 than our spectroscopic observations due to their longer time baseline. Therefore, during the pre-whitening of the spectroscopic data, we used the much more precisely known frequency values from photometry.

The first moment $\left\langle v^{1}\right\rangle$, representing the radial velocity, is dominated by the frequency $f_{1}=6.898 \mathrm{~d}^{-1}$ having an amplitude of $2.2 \mathrm{~km} \mathrm{~s}^{-1}$. The same situation occurs in the photometric data where $f_{1}$ has an amplitude of 27 mmag in the Stromgren $y$ filter and all other frequencies have amplitudes lower than half of this value. All other frequencies that are present in the first moment were also detected in the photometric data. In fact, we detected all but one non-combination frequency $\left(f_{13}=12.6967 \mathrm{~d}^{-1}\right)$ in the spectroscopic data having amplitudes down to $120 \mathrm{~m} \mathrm{~s}^{-1}$. The frequency separation of $0.02 \mathrm{~d}^{-1}$ between $f_{6}$ and $f_{9}$ is lower than the theoretical frequency resolution $0.05 \mathrm{~d}^{-1}$ of our data. Nevertheless, after prewhitening of $f_{6}$ a significant peak remains close-by, and pre-whitening with the frequency value of $f_{9}$, which is known from photometry, successfully removes this peak. 
Table 4. Pulsation frequencies and amplitudes of 44 Tau detected by Fourier analysis of the first $\left(\left\langle v^{1}\right\rangle\right)$ and second $\left(\left\langle v^{2}\right\rangle\right)$ moment, the pixel-bypixel amplitude, and photometric data (A07). The frequency values are taken from A07. $A_{\left\langle v^{1}\right\rangle}$ denotes the amplitude of the first moment; $S N R_{\left\langle v^{1}\right\rangle}$ is the corresponding signal-to-noise ratio; $A_{\left\langle v^{2}\right\rangle}$ is the amplitude of the second moment and $A_{P b P}$ is the amplitude of the pixel-intensity variations integrated across the line. The photometric amplitudes $A_{v}$ and $A_{y}$ are from the observing season 2002/03 (A07).

\begin{tabular}{lccccccc}
\hline \hline ID & $\begin{array}{c}\text { freq. } \\
\mathrm{d}^{-1}\end{array}$ & $\begin{array}{c}A_{\left\langle v^{1}\right\rangle} \\
\mathrm{km} \mathrm{s}^{-1} \\
\pm 0.03\end{array}$ & $S N R_{\left\langle v^{1}\right\rangle}$ & $\begin{array}{c}A_{\left\langle v^{2}\right\rangle} \\
\left(\mathrm{km} \mathrm{s}^{-1}\right)^{2}\end{array}$ & $\begin{array}{c}A_{P b P} \\
\mathrm{~km} \mathrm{~s}^{-1}\end{array}$ & $\begin{array}{c}A_{v} \\
\mathrm{mmag} \\
\pm 0.17\end{array}$ & $\begin{array}{c}A_{y} \\
\mathrm{mmag} \\
\pm 0.13\end{array}$ \\
\hline$f_{1}$ & 6.8980 & 2.22 & 100.5 & - & 1.58 & 39.52 & 27.05 \\
$f_{2}$ & 7.0060 & 0.46 & 21.2 & - & 0.41 & 14.01 & 9.01 \\
$f_{3}$ & 9.1175 & 0.44 & 24.3 & $(1.85)^{1}$ & 0.42 & 17.85 & 11.99 \\
$f_{4}$ & 11.5196 & 0.73 & 40.6 & $(1.98)^{1}$ & 0.58 & 16.43 & 11.40 \\
$f_{5}$ & 8.9606 & 1.03 & 55.6 & - & 0.67 & 13.12 & 8.85 \\
$f_{6}$ & 9.5613 & 0.30 & 15.9 & - & - & 20.56 & 13.67 \\
$f_{7}$ & 7.3034 & 0.70 & 33.1 & - & 0.38 & 7.50 & 5.38 \\
$f_{8}$ & 6.7953 & 0.28 & 12.6 & - & 0.26 & 3.76 & 2.83 \\
$f_{9}$ & 9.5801 & 0.12 & 6.4 & - & - & 4.65 & 3.12 \\
$f_{10}$ & 6.3390 & 0.21 & 8.5 & - & - & 3.11 & 1.99 \\
$f_{11}$ & 8.6394 & 0.32 & 17.1 & - & 0.23 & 1.61 & 1.38 \\
$f_{12}$ & 11.2946 & 0.11 & 5.8 & - & - & 1.28 & 1.18 \\
$2 * f_{1}$ & 13.7962 & 0.08 & 3.8 & 2.47 & 0.24 & 1.02 & 0.61 \\
$f_{1}+f_{5}$ & 15.8586 & 0.10 & 4.6 & 2.26 & 0.22 & 1.01 & 0.91 \\
$f_{1}+f_{3}$ & 16.0155 & 0.09 & 4.0 & 2.41 & 0.20 & 0.85 & 0.79 \\
$f_{1}+f_{4}$ & 18.4176 & - & - & - & 0.20 & 1.18 & 1.11 \\
\hline
\end{tabular}

${ }^{1}$ These frequencies have relatively low signal-to-noise ratios of $S N R_{f 3}=3.0, S N R_{f 4}=3.6$.

We also detected the first harmonic of $f_{1}$ which can be attributed to the non-sinusoidal shape of the first moment variations. Furthermore, two combination frequencies $f_{1}+f_{5}$ and $f_{1}+f_{3}$ have significant amplitudes. The other combination frequencies, known from photometry, could not be detected, presumably due to the fact that their amplitudes lie below the significance limit. In total, we detected 15 frequencies in the first moment variations of our combined line profile.

These spectroscopic data demonstrate very nicely the importance of obtaining photometric measurements for determining the frequency values with high precision. Mainly due to aliasing effects, correct determination of $f_{9}, f_{10}$ and $f_{12}$ would have been very difficult, if not impossible, from the spectroscopic data alone (see Fig. 4). After prewhitening with these terms the residuals lie far below the significance limit.

The analysis of the second moment $\left\langle v^{2}\right\rangle$, a measure of the width variations of the line profile, revealed $2 f_{1}, f_{1}+f_{5}$ and $f_{1}+f_{3}$. Two frequencies, $f_{3}$ and $f_{4}$, that were also detected in $\left\langle v^{1}\right\rangle$, appear in the second moment but with low significance. Generally speaking, axisymmetric pulsation modes have their dominant term $2 f$ in the second moment, while $m \neq 0$ tesseral modes have both $f$ and $2 f$, and $\ell=m$ modes have only $f$ as dominant term in the second moment (Aerts et al. 1992). Therefore, we are apparently dealing with a star that exhibits a large number of axisymmetric modes. This would indicate a low inclination angle which prefers the detection of axisymmetric modes due to geometric cancellation of the non-axisymmetric modes. Such a situation is present in the $\delta$ Sct star FG Vir (Zima et al. 2006) and accounted for a selection effect due to the low inclination angle of only $19^{\circ}$. We will focus on the mode identification in more detail in Sect. 4.2.

A second approach to search for periodicities was carried out by computing a separate Fourier analysis for every single wavelength bin across the line profile and averaging the resulting Fourier spectra. This analysis revealed only eight of the previously known independent frequencies and four combination frequencies. Among the latter is one term, which was not revealed in the first moment: $f_{1}+f_{4}=18.4176 \mathrm{~d}^{-1}$.

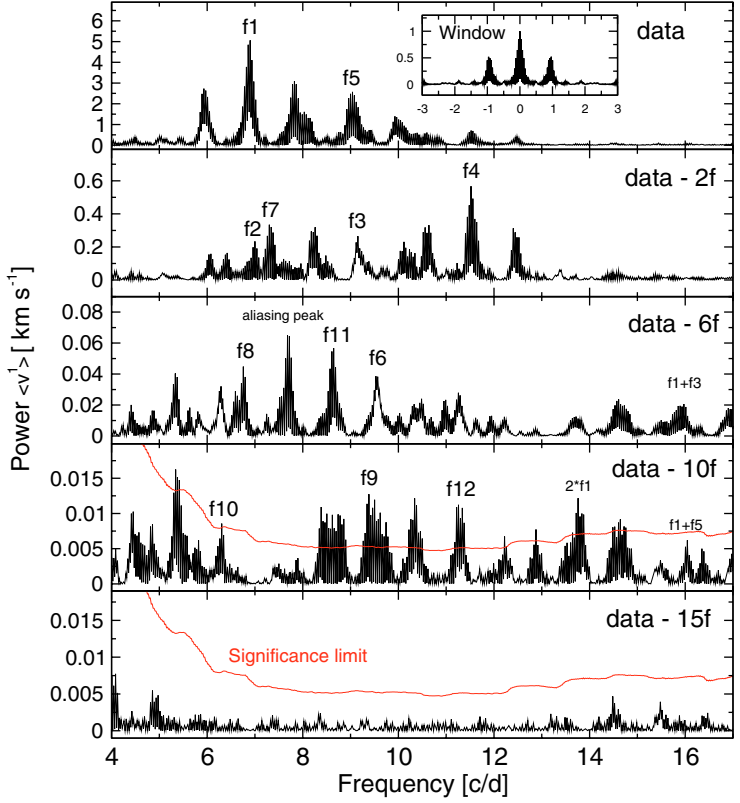

Fig. 4. Fourier analysis of the first moment of the combined line profile (described in the text). The top most panel shows the Fourier spectrum of the original data and each subsequent lower panel is prewhitened with the above-indicated frequencies by least-squares fitting. The bottom panel shows the residuals which are clearly below the significance limit. The latter is a running mean of 11.8 times the noise in power (Breger et al. 1996).

The spectroscopically detected frequencies and their amplitudes are listed in Table 4 . The frequency numbering is identical to the convention used in A07.

\subsection{Mode identification}

For objects like $\delta$ Sct and $\beta$ Cep stars which pulsate in the nonasymptotic regime with low-radial order $p$-modes, successful 


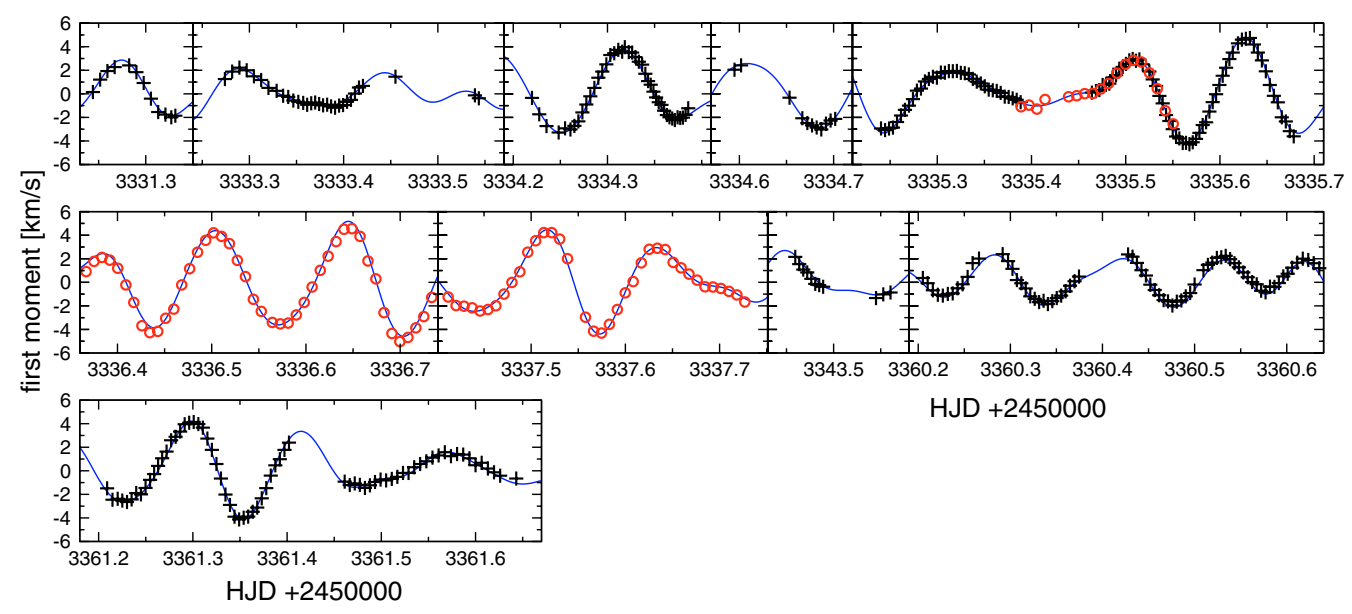

Fig. 5. Observations and multi-periodic 15-frequency fit (solid line) of the first moment variations of 44 Tau (o TLS, + NOT).

applications of asteroseismology are very sparse. For these stars, we do not only require a large number of observed pulsation frequencies but it is also necessary to assign to the observed frequencies pulsational quantum numbers, $\ell$ and $m$, to enable a qualitative comparison with theoretical seismic models. Such mode identifications have been successfully carried out for a large number of $\beta$ Cep stars (see, e.g. Aerts $\&$ de Cat 2003) and $\delta$ Sct stars (see, e.g. Mantegazza 2000) adopting spectroscopic identification methods that enable the determination of $\ell, m$, the pulsational amplitude and the stellar inclination angle.

Due to the low rotational broadening of the absorption lines we cannot apply mode identification methods that rely on Doppler broadening. This excludes the pixel-by-pixel method, the intensity-period-search method, and the Fourier parameter fit method. All these methods make use of the temporal intensity variations across the line profile. Due to the low $v \sin i$ of 44 Tau, these methods are especially not well suited for determining the azimuthal order $m$. Moreover, the amplitude of the dominant pulsation frequency of 44 Tau is of the same order as $v \sin i$. It has been shown by Zima et al. (2004b) that in such a case the amplitude and phase across the line of the other pulsation frequencies can be severely distorted, which hampers their identification.

Figure 6 shows zeropoint, amplitude and phase across the combined line profile for pulsation frequencies detected from the pixel-intensity variations. It is evident that the double-peaked behavior of the amplitude along the profile is very similar for all independent pulsation modes and the phase shows in most cases a phase jump of $\pi$ at the line center - an indicator for axisymmetric $p$-mode pulsation. The harmonic $2 f_{1}$ and the three combination frequencies have maximum amplitude at the line center and a $2 \pi$ phase shift across the line. Such a behavior of harmonic/combination frequencies was also reported by Zima et al. (2006) for the star FG Vir.

For slowly rotating stars the moment method (Briquet \& Aerts 2003) is very well suited since it does not rely on rotational Doppler broadening of the line profile. By means of this method, the time-series of the first three moments of the observed line profile are fitted with synthetic moments. This method's high computational speed makes it possible to search through a large parameter space and thus to test many different solutions. Drawbacks are its inability to determine the statistical significance of a fit which often prevents selection between different solutions and the fact that a maximum of six independent frequencies can be fitted simultaneously. This limit is set by the complexity of the frequency coupling of the moments
(Mathias et al. 1994). Therefore, since 12 independent frequencies are present in the first moment of 44 Tau, we could not apply the moment method to all observed pulsation frequencies simultaneously.

In a first approach we applied the moment method to six frequencies at a time, neglecting the six other frequencies which are present in the moments. In order to keep the effect of the six ignored frequencies on the fitting as small as possible, we fitted the two dominant frequencies $f_{1}$ and $f_{5}$ together with four other modes. Nevertheless, this approach did not yield satisfactory constraints on the mode parameters and it was not possible to select between more than 20 possible solutions. The reason therefore is the fact that the frequencies neglected during the fitting are actually treated as noise in the moment time-series. This increased noise level in turn increased the ambiguity of the solutions.

A better suited approach to apply the moment method to 44 Tau was described and tested in Zima et al. (2004a). For each of the eight frequencies, detected from the temporal intensity variations across the profile we computed 20 phase bins for the period of the mode under consideration. By doing so, all other pulsation modes are averaged out and we deal essentially with time-series of mono-mode pulsations. For each frequency we then separately applied the moment method assuming monomode pulsation. The following parameter space was searched [min, max, step]: $\ell=[0,3,1], m=[-\ell, \ell, 1]$ for each $\ell, i=$ $\left[5^{\circ}, 85^{\circ}, 5^{\circ}\right], v \sin i=[0,20,0.1] \mathrm{km} \mathrm{s}^{-1}, \sigma=[1,15,0.1] \mathrm{km} \mathrm{s}^{-1}$.

The results of the moment method are summarized in Table 5. For each pulsation frequency the four best solutions having the lowest value of the discriminant $\Gamma$ from the application of the moment method are listed. Although, the discriminants of the best solutions are quite close and a statistical criterion does not exist to select between different solutions (see, e.g., De Ridder et al. 2005), for most modes the azimuthal order $m$ can be estimated. Accordingly, $f_{1}, f_{4}, f_{5}, f_{7}, f_{8}$, and $f_{11}$ have with high probability axisymmetric character. For two of the analyzed frequencies the best solutions identify them as prograde pulsation modes ${ }^{1}$ with $m=1: f_{2}$ and $f_{3}$.

The harmonic degree $\ell$ cannot be constrained for any of the pulsation frequencies. Several theoretical and observational studies carried out with the moment method have revealed that generally more than one solution can explain the observations

\footnotetext{
${ }^{1}$ In our formalism the temporal dependence of the displacement field is given by $\mathrm{e}^{-\mathrm{i} \omega t}$.
} 

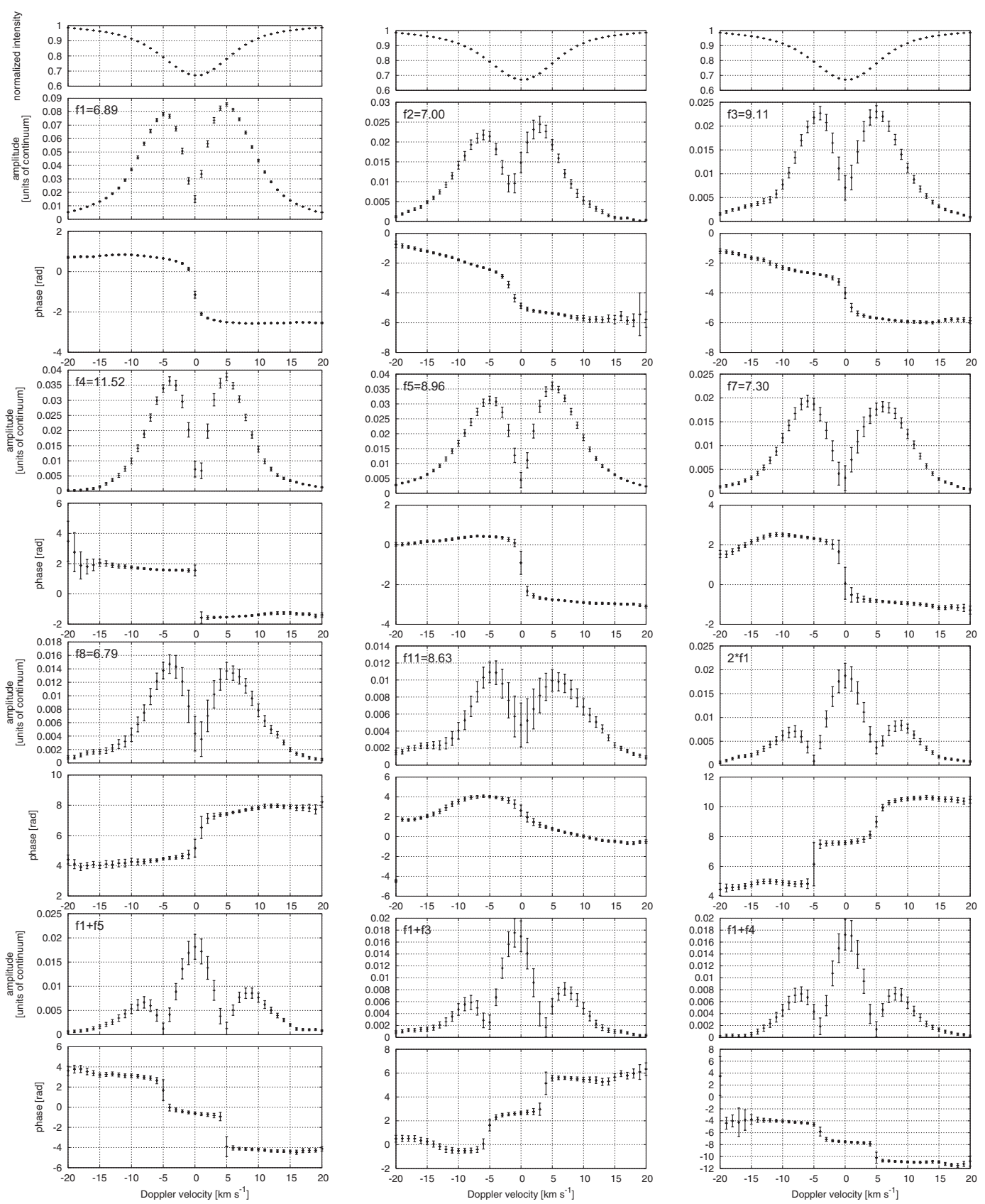

Fig. 6. Fourier parameters across the combined line profile (see text) of the detected pulsation frequencies. The top panels show the zero-point profile. For every single frequency, the observed amplitude in units of the continuum and the phase distribution in radians is shown.

(see, e.g. De Pauw et al. 1993; Mathias et al. 1993; Briquet \& Aerts 2003). Furthermore, their applications of the moment method to slowly rotating stars show a similar behavior of the ambiguities, namely that $m$ is generally better constrained than $\ell$. A possible explanation my lie in the fact that the second moment is a very sensitive discriminator between axisymmetric $(m=0)$ and non-axisymmetric modes $(m \neq 0)$. So, it is no surprise that our identification presented in Table 5 shows these ambiguities, especially in the identification of $\ell$.

The determined values of $v \sin i$ cover a large range between 1 and $16 \mathrm{~km} \mathrm{~s}^{-1}$ and are for most modes much higher than the value of $2 \mathrm{~km} \mathrm{~s}^{-1}$ that we determined from line synthesis. The reason therefore is apparently a combination of two effects. The moment method assumes a Gaussian intrinsic profile whereas the observed profiles clearly have a Voigtian shape and thus much broader wings. Moreover, the phase averaging we carried out to separate each pulsation mode cannot eliminate the pulsational broadening induced by the other modes. This may also lead to an overestimation of $v \sin i$ and $\sigma$.

Axisymmetric pulsation modes are not well suited for determining the inclination angle due to the interdependency of moment amplitude and inclination. Therefore, we should only take the inclination derived from $f_{2}$ and $f_{3}$ into account. Unfortunately, the fits to these two modes yield quite a large range of possible inclination values of between 35 and $85^{\circ}$. Taking into account the previously derived $v \sin i=2 \pm 1 \mathrm{~km} \mathrm{~s}^{-1}$, this implies for the equatorial rotational velocity a possible range between 1 and $5 \mathrm{~km} \mathrm{~s}^{-1}$. This results in a rotational splitting of between 0.006 and $0.027 \mathrm{~d}^{-1}$. Thus, the two frequencies $f_{6}$ and $f_{9}$ which are separated by $0.0188 \mathrm{~d}^{-1}$ could be rotationally split pulsation modes. 
Table 5. Mode parameters derived from the application of the moment method. For each pulsation mode, the four best solutions are shown. $\Gamma$ is the discriminant, $v$ is the velocity amplitude in $\mathrm{km} \mathrm{s}^{-1} ; i$ is the stellar inclination angle in degrees; $v \sin i$ is the projected rotational velocity, $\sigma$ is the width of the intrinsic Gaussian profile, both expressed in $\mathrm{km} \mathrm{s}^{-1}$.

\begin{tabular}{|c|c|c|c|c|c|c|}
\hline $\bar{\Gamma}$ & $\ell$ & $m$ & $v$ & $i$ & $v \sin i$ & $\sigma$ \\
\hline \multicolumn{7}{|c|}{$f_{1}=6.89 \mathrm{~d}^{-1}$} \\
\hline 3.16 & 1 & 0 & 8.2 & 15 & 15.4 & 1.5 \\
\hline 3.23 & 0 & 0 & 11.3 & - & 11.7 & 5.0 \\
\hline 3.27 & 2 & 0 & 9.3 & 5 & 11.5 & 5.0 \\
\hline 3.56 & 2 & 1 & 30.6 & 15 & 1.0 & 5.7 \\
\hline \multicolumn{7}{|c|}{$f_{2}=7.00 \mathrm{~d}^{-1}$} \\
\hline 3.22 & 1 & 1 & 2.2 & 85 & 14.8 & 3.2 \\
\hline 3.24 & 2 & 1 & 6.1 & 75 & 16.1 & 1.0 \\
\hline 3.26 & 3 & 0 & 7.0 & 25 & 7.2 & 6.8 \\
\hline 3.27 & 2 & 0 & 7.5 & 45 & 9.2 & 6.3 \\
\hline \multicolumn{7}{|c|}{$f_{3}=9.11 \mathrm{~d}^{-1}$} \\
\hline 3.26 & 3 & 1 & 7.5 & 35 & 3.0 & 7.5 \\
\hline 3.29 & 2 & 1 & 7.3 & 75 & 3.8 & 7.5 \\
\hline 3.32 & 1 & 1 & 4.4 & 35 & 4.5 & 7.4 \\
\hline 3.33 & 3 & 3 & 10.5 & 65 & 2.0 & 7.5 \\
\hline \multicolumn{7}{|c|}{$f_{4}=11.52 \mathrm{~d}^{-1}$} \\
\hline 3.08 & 2 & 0 & 6.2 & 35 & 11.1 & 5.6 \\
\hline 3.10 & 3 & 0 & 6.5 & 5 & 8.8 & 6.4 \\
\hline 3.13 & 1 & 0 & 5.8 & 65 & 16.0 & 1.1 \\
\hline 3.28 & 0 & 0 & 3.4 & - & 16.0 & 1.2 \\
\hline \multicolumn{7}{|c|}{$f_{5}=8.96 \mathrm{~d}^{-1}$} \\
\hline 3.00 & 2 & 0 & 4.9 & 15 & 8.3 & 6.6 \\
\hline 3.01 & 1 & 0 & 8.4 & 65 & 11.9 & 5.2 \\
\hline 3.03 & 0 & 0 & 5.0 & - & 14.2 & 3.7 \\
\hline 3.20 & 3 & 0 & 8.9 & 5 & 6.3 & 6.9 \\
\hline \multicolumn{7}{|c|}{$f_{7}=7.30 \mathrm{~d}^{-1}$} \\
\hline 3.09 & 2 & 0 & 9.3 & 45 & 11.0 & 5.5 \\
\hline 3.10 & 3 & 0 & 5.4 & 15 & 9.4 & 6.2 \\
\hline 3.26 & 1 & 0 & 7.4 & 75 & 16.0 & 1. \\
\hline 3.30 & 3 & 1 & 19.0 & 55 & 7.4 & 6.3 \\
\hline \multicolumn{7}{|c|}{$f_{8}=6.79 \mathrm{~d}^{-1}$} \\
\hline 3.05 & 3 & 0 & 5.8 & 65 & 11.2 & 5.6 \\
\hline 3.06 & 2 & 0 & 6.0 & 65 & 10.2 & 6.0 \\
\hline 3.08 & 3 & -1 & 4.6 & 25 & 1.2 & 7.7 \\
\hline 3.08 & 2 & -1 & 2.9 & 35 & 1.9 & 7.7 \\
\hline \multicolumn{7}{|c|}{$f_{11}=8.63 \mathrm{~d}^{-1}$} \\
\hline 3.36 & 2 & 0 & 1.1 & 5 & 16.0 & 1.1 \\
\hline 3.37 & 3 & 0 & 2.3 & 5 & 10.5 & 5.9 \\
\hline 3.41 & 3 & 1 & 9.8 & 55 & 7.9 & 6.6 \\
\hline 3.43 & 2 & 1 & 10.8 & 85 & 12.1 & 5.0 \\
\hline
\end{tabular}

\section{Discussion and conclusions}

We acquired high signal-to-noise high-resolution time-series Echelle-spectra of the $\delta$ Sct star 44 Tau with the goal to determine the stellar and pulsational characteristics of this object. According to our abundance analysis, 44 Tau basically shows a solar abundance pattern. The derived atmospheric parameters of $T_{\text {eff }}=7000 \pm 200 \mathrm{~K}$ and $\log g=3.6 \pm 0.1$ imply that this is a well evolved object. At this evolutionary status $g$-modes have already penetrated the $p$-mode frequency domain by avoided crossing (Pamyatnykh, priv. comm.) which severely complicates the frequency spectrum. On the other hand, these frequencies yield important information about the deep stellar interior, where they propagate. A reliable mode identification is necessary to enable a meaningful comparison between observed and modeled frequency values.
Table 6. Best identifications of the azimuthal order $m$ of the spectroscopically detected pulsation modes of 44 Tau from the application of the moment method. The two modes identified by A07 from photometry as radial are indicated in the last column.

\begin{tabular}{cccc}
\hline \hline name & $\begin{array}{c}\text { frequency } \\
\mathrm{d}^{-1}\end{array}$ & $\begin{array}{c}m \\
\text { this work }\end{array}$ & $\begin{array}{c}\ell \\
\text { from A07 }\end{array}$ \\
\hline$f_{1}$ & 6.8980 & 0 & 0 \\
$f_{2}$ & 7.0060 & 1 & - \\
$f_{3}$ & 9.1175 & 1 & - \\
$f_{4}$ & 11.5196 & 0 & - \\
$f_{5}$ & 8.9606 & 0 & 0 \\
$f_{7}$ & 7.3034 & 0 & - \\
$f_{8}$ & 6.7953 & 0 & - \\
$f_{11}$ & 8.6394 & 0 & - \\
\hline
\end{tabular}

We carried out an identification of eight pulsation frequencies by applying the moment method and were able to constrain the azimuthal order $m$ of all modes. Accordingly, six frequencies are axisymmetric and two are prograde with $m=1$. The degree $\ell$ could not be constrained due to ambiguities in the mode identification regarding this parameter. According to A07, two radial pulsation modes have been identified, the fundamental, $f_{1}$ and the first overtone, $f_{5}$. The identification of these two modes alone sets already strong constraints on the evolutionary status and the metal abundance.

The star 44 Tau is one of the main photometric targets of the Delta Scuti Network and additional photometric data for 44 Tau for the time period 2004 through 2008 will become available in the next few years. The 2004 data were taken simultaneously with the spectroscopic data reported in the present paper. Consequently, photometric determinations of the $\ell$ value can be expected for additional modes. Our identification of the azimuthal order yields important complementary information. We determined a very low value of the projected equatorial rotation velocity of $2 \pm 1 \mathrm{~km} \mathrm{~s}^{-1}$. Considering the range determined for the stellar inclination angle, the resulting equatorial rotational velocity of 44 Tau is $3 \pm 2 \mathrm{~km} \mathrm{~s}^{-1}$. Consequently, rotational effects of higher order may be neglected during the seismic modeling. Due to this fact, the excellent observational photometric/spectroscopic data and the provided mode identification, this object is a very promising target for successful asteroseismic modeling.

Acknowledgements. This work was supported by the Austrian Fonds zur Förderung der wissenschaftlichen Forschung (Projects P17441-N02 and P18224-N13). W.Z. is supported by the Research Council of Leuven University, under grant GOA/2003/04. W.Z. wants to thank Katrien Kolenberg and Conny Aerts for many inspiring discussions and Maarten Desmet for the help with the moment method software.

\section{References}

Aerts, C. 1996, A\&A, 314, 115

Aerts, C., \& De Cat, P. 2003, SSRv, 105, 453

Aerts, C., De Pauw, M., \& Waelkens, C. 1992, A\&A, 266, 294

Antoci, V., Breger, M., Rodler, F., Bischof, K., \& Garrido, R. 2007, A\&A, 463, 225 (A07)

Balona, L. A. 1986, MNRAS, 219, 111

Breger, M. 2000, in Delta Scuti and Related Stars, ed. M. Breger, \& M. H. Montgomery, ASP Conf. Ser., 210, 3

Breger, M., Handler, G., Serkowitsch, E., et al. 1996, A\&A, 309, 197

Breger, M., Pamyatnykh, A. A., Pikall, H., \& Garrido, R. 1999, A\&A, 341, 151

Breger, M., Lenz, P., Antoci, V., et al. 2005, A\&A, 435, 955

Briquet, M., \& Aerts, C. 2003, A\&A, 398, 687

Danziger, I. J., \& Dickens, R. J. 1967, ApJ, 122, 2042

De Pauw, M., Aerts, C., \& Waelkens, C. 1993, A\&A, 280, 493

De Ridder, J., Molenberghs, G., \& Aerts, C. 2005, Appl. Stat., 65, 1 
Desikachary, K. 1973, A\&A, 27, 331

Daszynska-Daszkiewicz, J., Dziembowski, W. A., Pamyatnykh, A. A., et al. 2005, A\&A, 438, 653

Grevesse, N., \& Sauval, A. J. 1998, SSRv, 85, 161

Hauck, B., \& Mermilliod, M. 1998, A\&AS, 129, 431

Ilyin, I. V. 2000, High resolution SOFIN CCD Echelle spectroscopy, Ph.D. Thesis, University of Oulu

Kochukhov, O. 2006, in Magnetic Stars, ed. I. Romanyuk, \& D. Kudryavtsev

Kupka, F., Piskunov, N., Ryabchikova, T. A., Stempels, H. C., \& Weiss, W. W. 1999, A\&AS, 138, 119

Kurucz, R. L. 1993, CDROM13, SAO, Cambridge

Lenz, P., \& Breger, M. 2005, CoAst, 146, 53

Lopez de Coca, P., Rolland, A., Garrido, R., \& Rodriguez, E. 1987, Rev. Mex. Astron. Astrof., 15, 59

Loumos, G. L., \& Deeming, T. J. 1978, Ap\&SS, 56, 285

Mantegazza, L. 2000, in Delta Scuti and Related Stars, ed. M. Breger, \& M. H. Montgomery, ASP Conf. Ser., 210, 138

Mantegazza, L. 2004, A\&A, 415, 299

Mathias, P., Aerts, C., De Pauw, M., Gillet, D., \& Waelkens, C. 1993, A\&A, 283, 813

Mathias, P., Aerts, C., Gillet, D., \& Waelkens, C. 1994, A\&A, 289, 875

Nordstroem, B., Mayor, M., Andersen, J., et al. 2004, A\&A, 418, 989

Percy, J. R. 1973, The Observatory, 93, 81
Piskunov, N. E., Kupka, F., Ryabchikova, T. A., Weiss, W. W., \& Jeffery, C. S. 1995, A\&AS, 112, 525

Poretti, E., Mantegazza, L., \& Riboni, E. 1992, A\&A, 256, 113

Rodler, F., Breger, M., Zima, W., et al. 2003, Astrophysics and Space Science 284, No. 1 (Dordrecht: Kluwer Academic Publishers), 387

Rogers, N. Y. 1995, CoAst, 78

Ryabchikova, T. A., Piskunov, N. E., Stempels, H. C., Kupka, F., \& Weiss, W. W. 1999, Phys. Scr., T83, 162

Schrijvers, C., Telting, J. H., Aerts, C., Ruymaekers, E., \& Henrichs, H. F. 1997, A\&AS, 121, 343

Schrijvers, C., \& Telting, J. H. 2002, A\&A, 394, 603

Shulyak, D., Tsymbal, V., Stütz, Ch., Ryabchikova, T., \& Weiss, W. W. 2004, A\&A, 428, 993

Smith, M. A. 1982, ApJ, 254, 242

Solano, E., \& Fernley, J. 1997, A\&AS, 122, 131

Zima, W. 2006, A\&A, 455, 235

Zima, W., Kolenberg, K., Briquet, M., \& Breger, M. 2004a, CoAst, 144, 5

Zima, W., Kolenberg, K., Briquet, M., \& Breger, M. 2004b, The A-Star Puzzle, held in Poprad, Slovakia, July 8-13, 2004, ed. J. Zverko, J. Ziznovsky, S. J. Adelman, \& W. W. Weiss (Cambridge, UK: Cambridge University Press), IAU Symp., 224, 853

Zima, W., Wright, D., Bentley, J., et al. 2006, A\&A, 455, 235 